\title{
Effect of Prior Chronic Aerobic Exercise on Overload-Induced Skeletal Muscle Hypertrophy in Mice
}

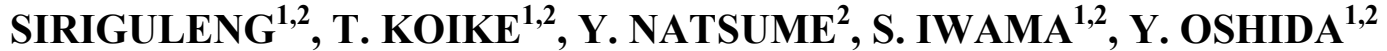 \\ ${ }^{1}$ Department of Sports Medicine, Graduate School of Medicine, Nagoya University, Nagoya, Japan, \\ ${ }^{2}$ Research Center of Health, Physical Fitness and Sports, Nagoya University, Nagoya, Japan
}

Received October 24, 2017

Accepted May 14, 2018

\section{Summary}

This study aimed to examine how regular aerobic training can affect the muscle hypertrophy induced by overloading. Male C57BL/6] mice were randomly divided into three groups: rest group, low-intensity aerobic exercise group, and high-intensity aerobic exercise group. Mice in the exercise groups were assigned to run at a speed of $10 \mathrm{~m} / \mathrm{min}$ (low-intensity) or $25 \mathrm{~m} / \mathrm{min}$ (high-intensity) for $30 \mathrm{~min} /$ day, five days/week, for four weeks. Then, the right hind leg gastrocnemius muscles were surgically removed to overload the plantaris and soleus muscles, while the left hind leg was subjected to a sham-operation. Both the plantaris and soleus muscles grew larger in the overloaded legs than those in the sham-operated legs. Muscle growth increased in the plantaris muscles in the low-intensity exercise group compared to that in the rest or high-intensity exercise groups at one and two weeks after overloading. This enhancement was not observed in the soleus muscles. Consistently, we observed changes in the expression of proteins involved in anabolic intracellular signaling, including Akt, mechanistic target of rapamycin (mTOR), and p70S6K, in the plantaris muscles. Our data showed for the first time that chronic low-intensity aerobic exercise precipitates overload-induced muscle growth.

\section{Key words}

Aerobic exercise $\bullet$ Skeletal muscle $\bullet$ Hypertrophy $\bullet$ mTOR protein

\section{Corresponding author}

T. Koike, Research Center of Health, Physical Fitness and Sports, Nagoya University, Nagoya 464-8601, Japan. Fax: 81-52-7893957. E-mail: koike@htc.nagoya-u.ac.jp

\section{Introduction}

Skeletal muscle is a critical organ for maintaining physical strength and metabolic function. The importance of maintaining muscle mass and function has gathered the attention of scientists in aging society. Sarcopenia, the age-related loss of muscle mass and strength, which is accompanied by accumulation of muscle fat, is the main cause of frailty among the elderly (Marcell 2003, Xue 2011), which has been recognized as the main medical issue in aging societies.

Physical exercise, together with nutrition, is the main intervention used for preventing and treating muscle loss (Dickinson et al. 2013). Exercise training can cause the molecular and metabolic remodeling of skeletal muscles (Egan et al. 2013). Although the effectiveness of high-intensity resistance exercise on the increase of muscle mass and strength has been established by many studies (Peterson et al. 2014), such exercise is difficult to sustain and may be risky for the elderly or people with chronic diseases. The benefits of aerobic training have been linked mostly to the resultant increases in endurance capacity and insulin sensitivity (Jiang et al. 2010). However, aerobic training has also been shown to alter protein metabolism and induce muscle hypertrophy (Fujita et al. 2007, Harber et al. 2010, Harber et al. 2009, Harber et al. 2009, Harber et al. 2012, Konopka et al. 2014, Short et al. 2004).

In the present study, we investigated the effect of chronic aerobic exercise on overload-induced skeletal muscle hypertrophy. We aimed to examine how regular aerobic training, which has multiple effects such as increasing insulin sensitivity (Yuan et al. 2013, Cho et al. 
2014) and suppressing chronic inflammation (Jung et al. 2013, Kwon et al. 2014), can alter the muscle growth induced by overloading. We examined whether insulinAKT-mechanistic target of rapamycin (mTOR)-p70S6K signaling pathway, one of the major pathways responsible for muscle protein synthesis, and muscle RING finger 1 (MuRF1) and Forkhead box O1 (FoxO1) expression, which regulates muscle protein breakdown, are affected during overloading by the prior aerobic exercise. Furthermore, we tested whether the intensity of aerobic exercise changes the effect of the prior aerobic training.

\section{Methods}

Animals

All experimental procedures were performed in accordance with the Guide for the Care and Use of Laboratory Animals of Nagoya University. C57BL/6J male mice ( 8 weeks of age) were obtained from Chubu Kagakushizai Co. Ltd. (Nagoya, Japan). Mice were housed individually and fed with standard chow (Oriental Yeast Co., Ltd., Tokyo, Japan) and water ad libitum. After a week of acclimation, the mice were randomly divided into three groups: the rest group, the lowintensity exercise group, and the high-intensity exercise group. The mice were maintained in a $12: 12 \mathrm{~h}$ reversal light-dark environment at $23{ }^{\circ} \mathrm{C}$.

\section{Overload-induced muscle hypertrophy}

Overload-induced muscle hypertrophy is the model used to examine molecular and cellular mechanisms that regulate muscle growth (Spangenburg 2009). The sequence of the overloading study procedure is shown in Figure 1. Mice were anesthetized during operation with sodium pentobarbital $(50 \mathrm{mg} / \mathrm{kg}$, intraperitoneally). Overload-induced muscle hypertrophy was induced in the right hind legs by surgical excision of gastrocnemius muscles from the Achilles' tendon to the belly of the muscle as described previously (Makanae et al. 2013, Serrano et al. 2008). This operation induces the compensatory growth of the soleus and plantaris muscles. An incision through the skin was made, and the Achilles tendon was exposed in the left hind legs (sham-operated), which were used as controls. After one week or two weeks of overloading, muscles and epididymal fat were dissected under anesthesia, and mice were sacrificed. The wet weight of muscles was measured, and then, the muscles were frozen with liquid nitrogen and stored at $-80{ }^{\circ} \mathrm{C}$ until analysis.

\section{Exercise protocol}

Mice were assigned to treadmill running exercise (SEEDS Inc., Nagoya, Japan) with $0^{\circ}$ inclination. Mice in the low-intensity exercise group were assigned to run at a speed of $10 \mathrm{~m} / \mathrm{min}$. Mice in the high-intensity exercise group were assigned to run at $10 \mathrm{~m} / \mathrm{min}$ initially with an increment of 2 (or 1 on the eighth training day) $\mathrm{m} / \mathrm{min}$ each training day to $25 \mathrm{~m} / \mathrm{min}$. Accordingly, mice in the highintensity exercise group were assigned to run at $25 \mathrm{~m} / \mathrm{min}$ for $30 \mathrm{~min}$ per day on the eighth training day and the following training days. When mice in the high-intensity exercise group could not continue running with the increased pace, the treadmill speed was decreased so that the mice could continue running. Mice in the exercise groups ran $30 \mathrm{~min}$ per day, five days a week, for four weeks until the day prior to the operation. The total exercise volume was $300 \mathrm{~m} /$ day and $750 \mathrm{~m} /$ day in the lowintensity group and the high-intensity group, respectively. Mice were not assigned to the exercise during overloading. To understand the effect of low-intensity or high-intensity aerobic exercise (Fig. 2, Table 1, Fig. 5d), muscles of mice in the exercise groups were dissected $24 \mathrm{~h}$ after the final exercise. The wet weight of muscles was measured, and then, the muscles were frozen with liquid nitrogen and stored at $-80{ }^{\circ} \mathrm{C}$ until analysis.

1 week overloading group Rest $(n=8)$, Low $(n=8)$, High $(n=6)$

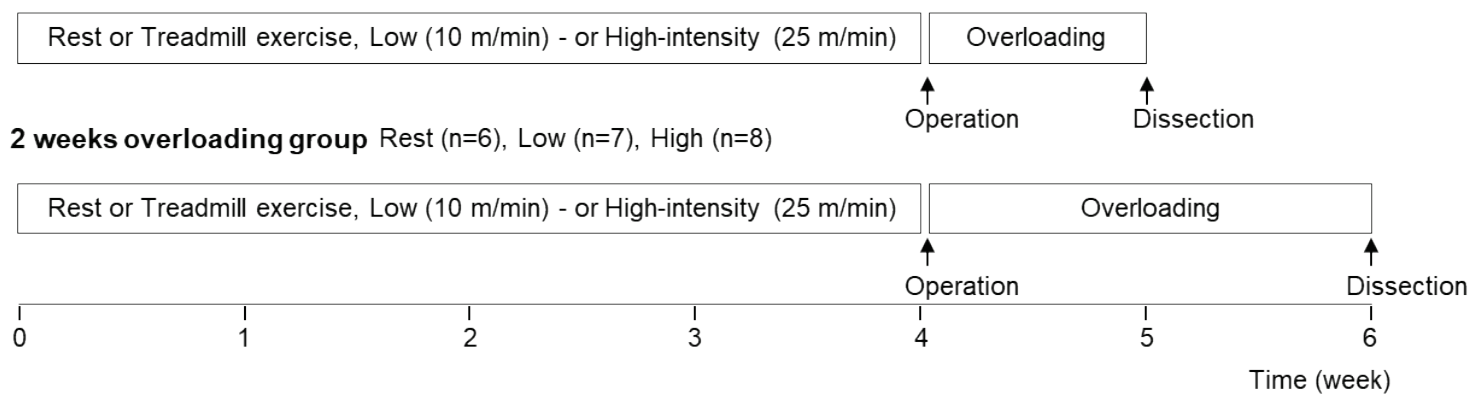

Fig. 1. Sequence of the study procedure for functional overloading. 


\section{Western blotting}

Western blotting was performed as described previously (Li et al. 2008). Briefly, $10 \mu \mathrm{g}$ of protein extracts from muscle were separated by SDS-PAGE at $20 \mathrm{~mA}$. The proteins were transferred to polyvinylidene difluoride (PVDF) membranes (EMD Millipore Corporation, Billerica, MA, USA) by semi-dry transfer at $25 \mathrm{~V}$ for $60 \mathrm{~min}$. After blocking membranes with $5 \%$ nonfat milk for one hour at room temperature, membranes were incubated overnight with a $1: 1,000$ dilution of the primary antibody at $4{ }^{\circ} \mathrm{C}$. The blots were then rinsed in PBS with $0.05 \%$ Tween 20 and incubated with a 1:1,000 dilution of the appropriate horseradish peroxidase-conjugated secondary antibody for $1 \mathrm{~h}$ at room temperature. Immunoreactive bands were detected using an ECL detection system (GE Healthcare UK Limited, Buckinghamshire, UK). Images of each membrane were taken on film and analyzed using Image-J software (National Institutes of Health, Bethesda, MD, USA). The individual rest/overload data points were divided by the group mean, thus the mean of the normalized rest/overload group is 1 with variability. The density of the protein band of the rest/sham-operated, low-intensity exercise/overload and sham-operated, and high-intensity exercise/overload and sham-operated groups was expressed as the fold change of the density of the rest/overload values.

Primary antibodies against phospho-Akt (Ser473), phospho-mTOR (Ser2448), total mTOR, phospho-p70S6K (Ser371), total p70S6K, total FoxO1, and total AMPK were obtained from Cell Signaling Technology (Beverly, MA, USA). Primary antibodies against total Akt1/2/3 and total MuRF1 were obtained from Santa Cruz Biotechnology, Inc. (Dallas, TX, USA). Horseradish peroxidase (HRP)-conjugated goat antirabbit (Bio-Rad, Laboratories Inc., Hercules, CA, USA) and anti-mouse (KPL, Gaithersburg, MD, USA) IgG antibodies were used as secondary antibodies.

\section{Statistical analysis}

All data were expressed as mean \pm S.D. The multiple group comparisons were made by one-way analysis of variance (ANOVA) followed by Tukey's test. A two-way ANOVA analysis was initially performed for Figures 3, 4 and 5, but the interaction was found between the variables (the type of exercise and overloading/shamoperated). One-way ANOVA analysis was performed among the 6 groups (rest/overload or sham-operated, low-intensity exercise/overload or sham-operated, and high-intensity exercise/overload or sham-operated) followed by Tukey's test. Significance was accepted at $\mathrm{P}<0.05$. All analyses were performed using GraphPad Prism 6.0 (GraphPad Software Inc., La Jolla, CA, USA).

\section{Results}

Effect of low-intensity or high-intensity aerobic exercise on leg muscles

The effect of 4-week treadmill exercise on muscle weight and consequent protein anabolic signaling was examined (Fig. 2, Table 1). There were no significant differences in body weight, lower leg muscle weight, or epididymal fat weight among the groups. Total food intake was significantly higher in the high-intensity exercise group than in the rest group. The analysis of Akt-mTOR-p70S6K signaling showed increased Akt phosphorylation only in the low-intensity exercise group. mTOR and p70S6K phosphorylation and Akt, mTOR, and p70S6K protein levels were not different among the rest, low-intensity, and high-intensity exercise groups.

Effect of prior aerobic exercise on overload-induced muscle growth

The effect of prior 4-week treadmill exercise on the growth of overloaded muscles for one week or two weeks was examined. To evaluate the time course of muscle growth, we measured muscle weights at one week and two weeks of overloading. Overloaded muscles were significantly heavier than muscles from sham-operated leg muscles in all groups for both the soleus and plantaris muscles (Fig. 3). In addition, the plantaris muscles from the overloaded legs of mice in the low-intensity exercise group but not in the high-intensity exercise group was heavier than those of mice in the rest group at both one week and two weeks of overloading (Fig. 3a). Soleus muscle weight in the overloaded leg was significantly lower in the high-intensity exercise group than in the rest group at two weeks (Fig. 3b). Table 2 shows the changes in body weight, overloaded leg muscle weight, and epididymal fat weight after one week or two weeks of overloading. Body weight was not significantly different among groups. Total food intake was not different among groups. Epididymal fat weight was significantly lower in the low-intensity exercise and high-intensity exercise groups than in the rest group. 
a

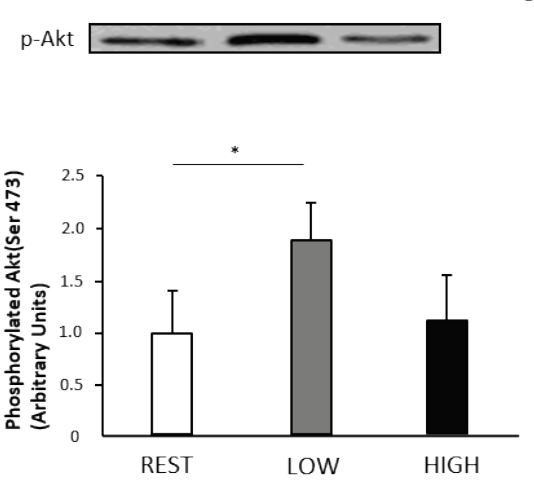
p-mTOR
p-p70S6K b

Total Akt

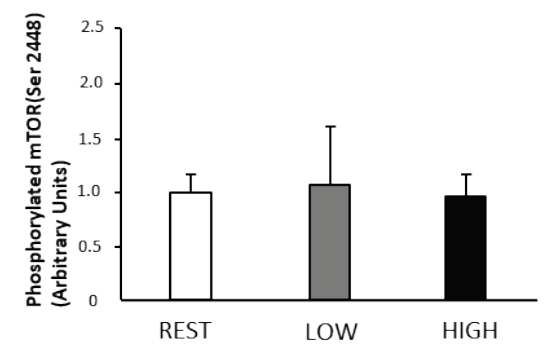

Total mTOR

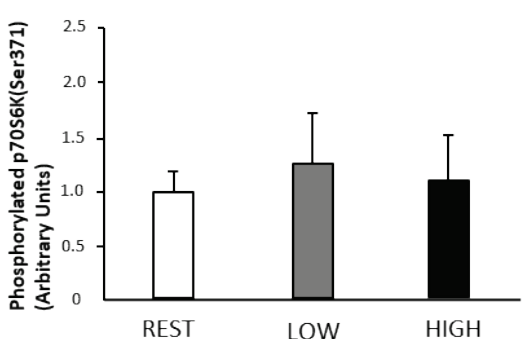

f Total p70S6K
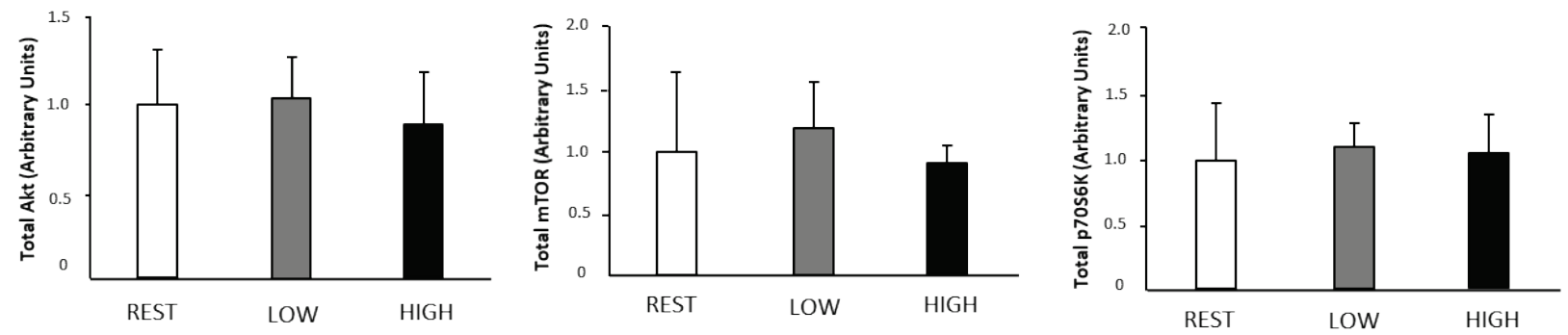

Fig. 2. Effect of aerobic exercise on the PI3K-Akt-mTOR pathway in the plantaris muscles. Phosphorylation and protein expression of Akt, mTOR, and p70S6K in the plantaris muscles after 4 weeks of aerobic exercise were analyzed by western blotting. Representative immunoblots are shown in the top panels. REST, rest group $(n=6)$; LOW, low-intensity exercise group ( $n=6)$; HIGH, high-intensity exercise group $(n=6)$. The comparison of data used ANOVA followed by Tukey's test. Data are expressed as mean \pm SD. The density of the protein band of the low-intensity and high-intensity exercise groups was expressed as the fold change of the density of the mean of the rest group values. Statistical difference vs. REST group $(* p<0.05)$.

Table 1. Body weight, weight of muscles, and epididymal fat weight after 4 weeks of aerobic exercise.

\begin{tabular}{lccc}
\hline & REST (n=6) & LOW $(\mathbf{n = 6})$ & HIGH (n=6) \\
\hline Body weight $(g)$ & $24.4 \pm 1.3$ & $24.0 \pm 1.2$ & $23.2 \pm 0.1$ \\
Weight of muscle $(\mathrm{mg})$ & & & \\
Gastrocnemius & $140 \pm 10$ & $137 \pm 9$ & $135 \pm 11$ \\
Plantaris & $23.8 \pm 2.3$ & $22.4 \pm 1.9$ & $22.0 \pm 2.0$ \\
Soleus & $11.6 \pm 1.5$ & $10.4 \pm 1.0$ & $10.3 \pm 1.4$ \\
Tibialis anterior & $49.0 \pm 4.1$ & $46.8 \pm 3.1$ & $44.5 \pm 3.6$ \\
Extensor digitorum longus & $11.5 \pm 0.8$ & $10.9 \pm 0.8$ & $11.1 \pm 0.7$ \\
Epididymal fat weight (mg) & $349 \pm 71$ & $356 \pm 73$ & $316 \pm 81$ \\
Total food intake (g/day) & $3.25 \pm 0.19$ & $3.31 \pm 0.32$ & $3.48 \pm 0.23 *$ \\
\hline
\end{tabular}

REST: rest group; LOW: low-intensity exercise group; HIGH: high-intensity exercise group. Data are expressed as mean \pm SD. Statistical difference vs. the REST group $(* p<0.05)$.

Effect of prior aerobic exercise on overload-induced anabolic signaling in muscles

To evaluate the time course of the signaling related to muscle protein synthesis, we examined the muscles dissected at one week and two weeks of overloading. Phosphorylation and protein expression of Akt, mTOR, and p70S6K were examined (Fig. 4). Phosphorylation and expression of Akt were increased in the overloaded legs compared to those in the shamoperated legs. Phosphorylation of Akt in the overloaded 
a

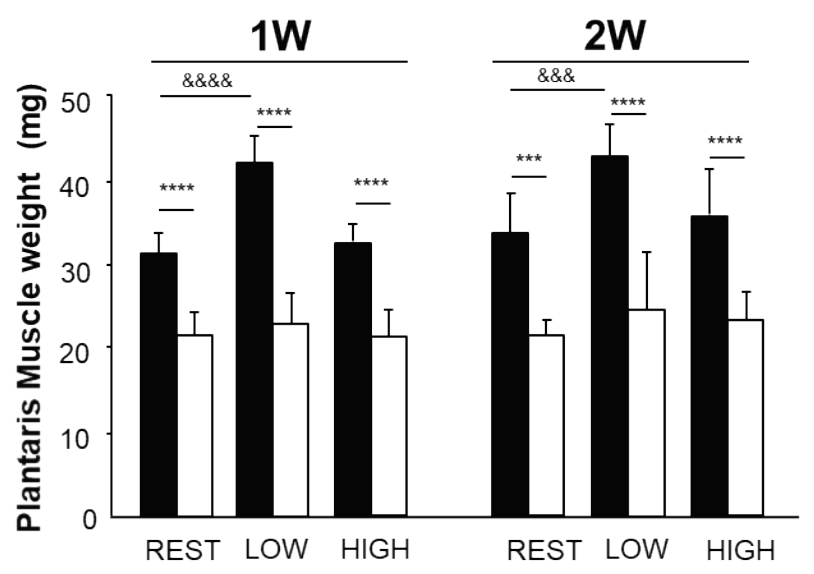

b

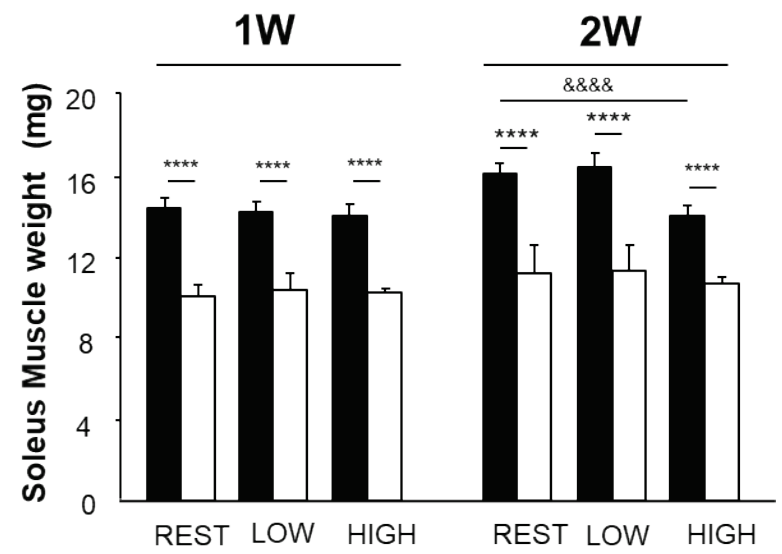

Overloaded legs

Sham-operated legs

Fig. 3. Effect of prior 4 weeks of aerobic exercise on muscle weight after 1 or 2 weeks of overloading. Weight of the plantaris muscle (a) and soleus muscle (b) of functionally overloaded legs (OL) or sham-operated legs (S) was measured after 1 or 2 weeks of overloading. REST, rest group ( 1 week: $n=8 ; 2$ weeks: $n=6$ ); LOW, low-intensity exercise group ( 1 week: $n=8 ; 2$ weeks: $n=7$ ); HIGH, high-intensity exercise group ( 1 week: $n=6 ; 2$ weeks: $n=8$ ). A significant difference was observed between OL and $S$ legs after 1 or 2 weeks of overloading $(* * * p<0.001, * * * * p<0.0001)$. The statistical analysis on the differences between 1 week and 2 weeks overloading were not made. The comparison of data from overloaded vs. sham operated leg used paired T-test. The comparison of data from different treatment groups used ANOVA followed by Tukey's test. Data are expressed as mean \pm SD. Statistical difference versus REST OL legs $\left({ }^{\& \& \&} p<0.001\right.$, \&\&\&\& $\left.p<0.0001\right)$.

Table 2. Body weight, weight of muscles, and epididymal fat weight after 1 week or 2 weeks of overloading.

\begin{tabular}{lcccccc}
\hline & \multicolumn{3}{c}{ 1 week of overloading } & \multicolumn{2}{c}{ 2 weeks of overloading } \\
\cline { 2 - 7 } & REST (n=8) & LOW (n=8) & HIGH (n=6) & REST (n=6) & LOW (n=7) & HIGH (n=8) \\
\hline Body weight $(g)$ & $25.4 \pm 1.7$ & $25.8 \pm 0.7$ & $25.5 \pm 1.6$ & $25.7 \pm 1.4$ & $25.7 \pm 1.8$ & $24.7 \pm 1.5$ \\
Weight of muscle $(\mathrm{mg})$ & & & & & & \\
Tibialis anterior & $46.2 \pm 2.6$ & $49.6 \pm 5.5$ & $46.2 \pm 3.9$ & $45.7 \pm 1.9$ & $48.6 \pm 2.4$ & $47.5 \pm 2.6$ \\
Extensor digitorum longus & $11.6 \pm 0.8$ & $11.3 \pm 0.5$ & $12.1 \pm 1.3$ & $11.6 \pm 0.5$ & $12.4 \pm 0.7$ & $11.6 \pm 0.7$ \\
Epididymal fat weight $(\mathrm{mg})$ & $354 \pm 27$ & $221 \pm 53^{* * * *}$ & $216 \pm 63^{* * *}$ & $413 \pm 11$ & $291 \pm 45^{*}$ & $281 \pm 56^{*}$ \\
Total food intake (g/day) & $3.64 \pm 0.12$ & $3.72 \pm 0.15$ & $3.73 \pm 0.10$ & $3.60 \pm 0.05$ & $3.65 \pm 0.11$ & $3.64 \pm 0.10$ \\
\hline
\end{tabular}

REST: rest group; LOW: low-intensity exercise group; HIGH: high-intensity exercise group. Data are expressed as mean \pm SD. \# Muscles of overloaded legs. Statistical difference vs. the REST group in each group. $(* p<0.05, * * * p<0.001, * * * * p<0.0001)$.

legs was significantly higher in the low-intensity exercise and high-intensity exercise groups than in the rest group after one week, but was lower after two weeks of overloading in high-intensity exercise group than in the rest group (Fig. 4a). Expression of Akt was significantly higher in the overloaded legs in the high-intensity exercise group than in the rest group after one week, but was lower after two weeks in the low-intensity exercise and high-intensity exercise groups than in the rest group (Fig. 4b).

Phosphorylation of mTOR was increased in overloaded legs compared to that in sham-operated legs. mTOR phosphorylation in the overloaded legs in the lowintensity exercise group but not in the high-intensity exercise group was significantly higher than that in the rest group after one week and two weeks of overloading (Fig. 4c). After one week of overloading, mTOR protein expression was increased in the overloaded legs compared to that in the sham-operated legs, with the exception of the low-intensity exercise group; mTOR protein expression in the overloaded legs in the low and high-intensity exercise groups was lower than that in the 


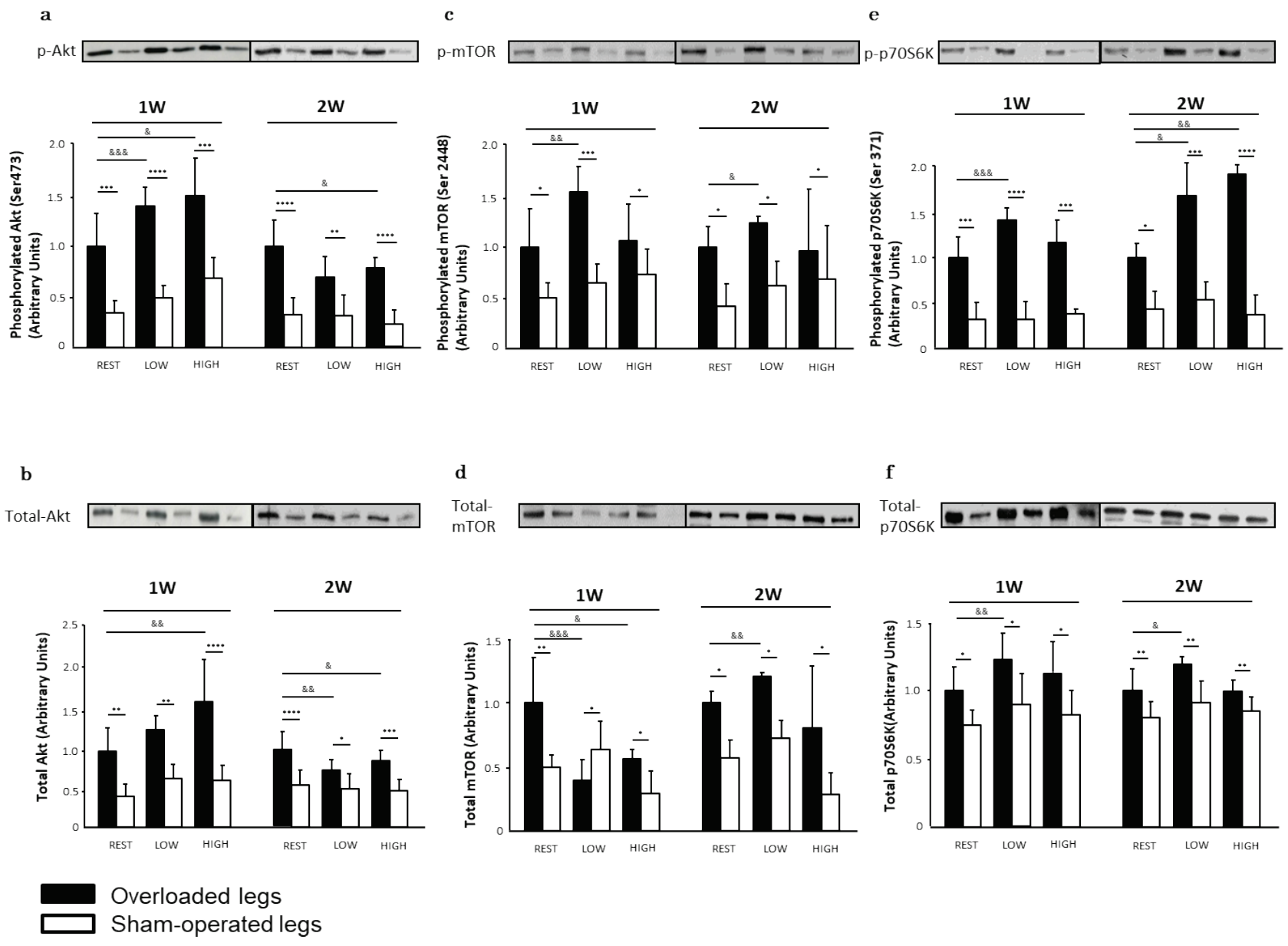

Fig. 4. Effect of prior 4 weeks of aerobic exercise on PI3K-Akt-mTOR pathway in the plantaris muscles, after overloading. Phosphorylation and protein expression of Akt, mTOR, and p70S6K in the plantaris muscles after 1 or 2 weeks of overloading were analyzed by western blotting. Representative immunoblots are shown at the top of the figures. REST, rest group ( 1 week: $n=8$; 2 weeks: $n=7)$; LOW, low-intensity exercise group ( 1 week: $n=8 ; 2$ weeks: $n=7)$; HIGH, high-intensity exercise group ( 1 week: $n=6$; 2 weeks: $n=8$ ); OL, overloaded legs; S, sham-operated legs; $1 \mathrm{~W}$, Overload of 1 week; $2 \mathrm{~W}$, Overload of 2 weeks. The statistical analysis on the differences between 1 week and 2 weeks overloading were not made. The density of the protein band of the lowintensity and high-intensity exercise groups was expressed as the fold change of the density of the mean of rest group (overloaded legs) values. Data are expressed as mean \pm SD. The comparison of data from overloaded vs. sham operated leg used paired T-test. The comparison of data from different treatment groups used ANOVA followed by Tukey's test. A significant difference was observed between $\mathrm{OL}$ and $\mathrm{S}$ legs $(* \mathrm{p}<0.05, * * \mathrm{p}<0.01, * * * \mathrm{p}<0.001, * * * * \mathrm{p}<0.0001)$. Significant differences vs. REST OL legs ( ${ }^{\&} \mathrm{p}<0.05$, \&\& $\left.p<0.01,{ }^{\text {\&\&\& }} p<0.001\right)$.

rest group. After two weeks of overloading, mTOR protein expression in the overloaded legs was increased compared to that in the sham-operated legs, and this expression in the overloaded legs in the low-intensity exercise group was increased compared to that in the rest group (Fig. 4d).

Phosphorylation and expression of p70S6K were increased in the overloaded legs compared to those in the sham-operated legs. Phosphorylation of p70S6K in the overloaded legs of the low-intensity exercise group was significantly higher than that in the rest group after one week and was significantly higher in the low- and highintensity exercise groups than that in the rest group after two weeks (Fig. 4e). Protein expression of p70S6K was higher in the low-intensity exercise group than in the rest group after one week and two weeks (Fig. 4f).

Effect of prior aerobic exercise on the protein expression of FoxOl and MuRF-1

Protein expression of FoxO1 was decreased in overloaded legs compared to that in sham-operated legs, and was lower in the overloaded legs in the low- or highintensity exercise groups than in the rest group after two weeks (Fig. 5a). Furthermore, expression of MuRF1 was lower in the overloaded legs compared to that in the shamoperated legs, and was lower in the overloaded legs in the low-intensity exercise and the high-intensity exercise groups than in the rest group after two weeks (Fig. 5b). 
a
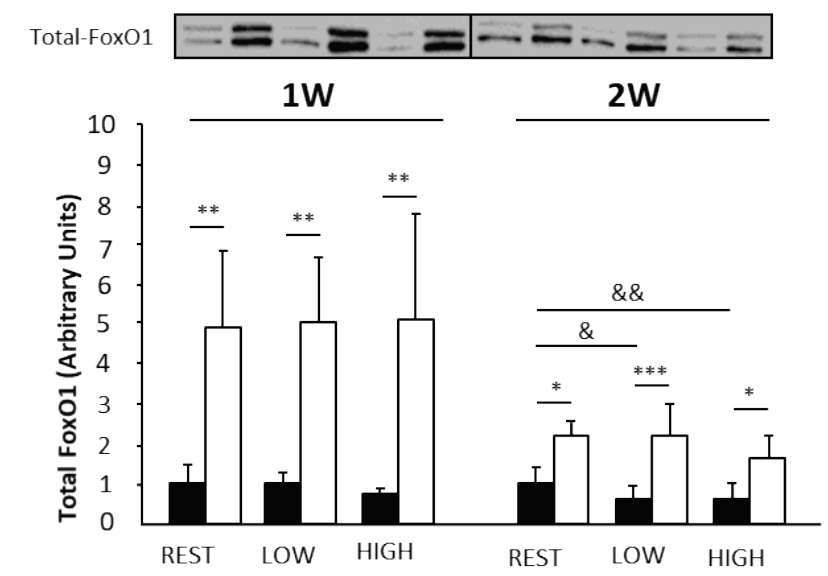

Total-AMPK $(\alpha)=-\infty-\infty-\infty \cdots \infty-\infty$



\section{b}


Overloaded legs

Sham-operated legs

Fig. 5. Effect of prior 4 weeks of aerobic exercise on FoxO1, MuRF1, and AMPK expression in the plantaris muscles, after overloading. Protein expression of FoxO1 (a), MuRF1 (b), and AMPK (c) after 1 or 2 weeks of overloading were analyzed by western blotting. Representative immunoblots are shown at the top of the figures. REST, rest group (1 week: $n=8 ; 2$ weeks: $n=7$ ); LOW, low-intensity exercise group ( 1 week: $n=8 ; 2$ weeks: $n=7$ ); $\mathrm{HIGH}$, high-intensity exercise group ( 1 week: $n=6 ; 2$ weeks: $n=8$ ); OL, overloaded legs; $\mathrm{S}$, sham-operated legs; $1 \mathrm{~W}$, Overload of 1 week; $2 \mathrm{~W}$, Overload of 2 weeks. The statistical analysis on the differences between 1 week and 2 weeks overloading were not made. The density of the protein band of the low-intensity and high-intensity exercise groups was expressed as the fold change of the density of the mean of rest group (overloaded legs) values. Protein expression of AMPK (d) after 4 weeks of aerobic training in the plantaris muscles were analyzed by western blotting. REST, rest group $(n=6)$; LOW, low-intensity exercise group $(n=6) ; H I G H$, high-intensity exercise group $(n=6)$; Data are expressed as mean \pm SD. The comparison of data from overloaded vs. sham operated leg used paired T-test. The comparison of data from different treatment groups used ANOVA followed by Tukey's test. The density of the protein band of the low-intensity and high-intensity exercise groups was expressed as the fold change of the density of the mean of the rest group values. Significant differences were observed between $\mathrm{OL}$ and $\mathrm{S}$ legs $(* \mathrm{p}<0.05, * * \mathrm{p}<0.01$, $* * * p<0.001)$, and vs. REST OL legs $\left({ }^{\&} p<0.05,{ }^{\text {\&\& }} p<0.01\right.$, \&\&\& $\left.p<0.001\right)$. Significant difference vs. REST group $\left({ }^{\$} p<0.05\right)$ in (d).

Effect of prior aerobic exercise on the expression of $A M P K$

Expression of AMP-activated kinase (AMPK) in the overloaded legs was lower than that in the shamoperated legs, and was lower in the overloaded legs in the low-intensity exercise group than in the rest group (Fig. 5c). AMPK expression was increased after four weeks of both the low-intensity and the high-intensity aerobic training (Fig. 5d).

\section{Discussion}

In this study, we showed that prior chronic aerobic training enhanced mechanical load-induced muscle hypertrophy. This effect was observed for lowintensity, but not high-intensity, aerobic training. To our knowledge, this study showed for the first time that chronic aerobic training can affect muscle growth induced by resistance stimuli. We believe that our data indicates the benefits of regular aerobic exercise. It is 
noteworthy that our results support the benefits of lowintensity exercise rather than high-intensity exercise.

The effect of aerobic training on skeletal muscle hypertrophy has yet to be established. Konopka et al. (2014) showed that aerobic exercise is effective for preventing age-related muscle loss through various mechanisms, including increased muscle protein synthesis (Harber et al. 2010, Harber et al. 2009, Harber et al. 2009, Short et al. 2004). Aerobic exercise has been shown to restore anabolic insulin signaling and increase protein synthesis in older adults (Fujita et al. 2007). In the present study, aerobic training itself did not affect muscle mass or mTOR-p70S6K signaling. AMPK is a serine/threonine kinase that is activated by intense exercise in an intensity-dependent manner (Egan et al. 2013). Continuous AMPK activation can decrease insulin resistance (Ruderman et al. 2013) and suppress chronic inflammation, which may enhance muscle growth.

Muscle mass is regulated by the balance between muscle protein synthesis and breakdown (Schiaffino et al. 2013). One of the reasons for decreased muscle mass in aging individuals is the anabolic resistance that occurs with age (Burd et al. 2013, Durham et al. 2010). mTOR has been shown to function as a signaling node that leads to muscle protein synthesis (Kennedy et al. 2016). Resistance exercise increases protein synthesis via three distinct signaling pathways initiated by insulin/insulinlike growth factor 1 (IGF1), mechanical loading, or amino acids (Kim et al. 2008). Activation of Akt precedes the activation of mTOR in the insulin/IGF1 pathway, but mechanical loading or amino acids can activate mTOR in an Akt-independent manner (Kim et al. 2008). The mTOR protein kinase is also found in two complexes: mTOR complex 1 (mTORC1) and mTOR complex 2 (mTORC2). The dynamic and complex changes in mTOR protein expression that occurred during our overloading experiment might be due to the interaction between diverse upstream signaling pathways and the involvement of the two distinct mTOR complexes, but we could not explain how this response of total-mTOR occurred. In the present study, however, the overall data on signaling is consistent with the increase of overload-induced muscle growth that occurred in the low-intensity exercise group.

The mTORC1-p70S6K pathway is required for protein synthesis in skeletal muscle. We found that prior high-intensity exercise did not increase overloadinginduced muscle growth. This is not consistent with the increase of p70S6K phosphorylation observed after two weeks of overloading, and might be due to the delayed activation of p70S6K phosphorylation in the highintensity exercise group compared with the activation of p70S6K in the low-intensity group. This difference in the profile of p70S6K may reflect the difference in AMPK activation between the low-intensity group and highintensity group, which suppress the mTOR-p70S6K pathway (Bolster et al. 2002). Finally, we examined the muscle protein degradation pathway. In catabolic conditions, activation of the degradation pathway contributes to muscle loss. FOXO transcription factors control the ubiquitin-proteasome system, and MuRF1 levels increase when the degradation pathway is activated (Sandri 2010). Expression of MuRF1, muscle-specific atrophy related ubiquitin ligase, was not different between the low-intensity and high-intensity exercise groups. The level of MuRF1 and FOXO1 expression seems to reflect the activation pattern of AKT in our study. AKT activation inhibits protein degradation by suppressing FOXO activity, which decreases MuRF1 expression (Sandri 2013). Our data show the degradation pathway related to MuRF1 is not activated, and does not explain our results showing the differential effect on muscle growth between the low-intensity and highintensity exercises.

In the present study, the hypertrophic effect of the prior low-intensity aerobic exercise was only observed in the plantaris muscle, a primarily type II muscle, but not in the soleus, a primarily type I muscle. Studies have shown that type II muscles are more sensitive to the effects of various physiological and pathological conditions than type I muscles (Holecek et al. 2017, Muthny et al. 2008, Koopman et al. 2006). The observed lack of effect of prior aerobic training on muscle growth or signaling in the soleus muscle (data not shown) may be due to the difference in susceptibility to overloading between type I and type II muscles, but it may also suggest that the mechanism underlying muscle growth (e.g. signaling pathway) differs between the plantaris and soleus muscles. Thomson et al. (2005) reported that AMPK activation diminished hypertrophy in aged fast-twitch (type II) skeletal muscle but not in slow-twitch (type I) soleus muscle. A previous study showed that the signaling proteins regulating hypertrophy may act differently between soleus and plantaris muscles (Gordon et al. 2001).

Recently, both aerobic training and resistant training are recommended for maintaining health (Garber et al. 2011). The optimal exercise mode, amount, and 
intensity for maintaining skeletal muscle have not been established. Although the present study suggests the benefit of low intensity aerobic training to muscle, we cannot address the combined effect of aerobic and resistance training on muscle mass because each exercise was introduced separately. Concurrent training, defined as simultaneous incorporation of endurance and resistance exercises, has been suggested to attenuate gains in muscle mass, strength, and power with resistant exercise alone (Fyfe et al. 2014). Lundberg et al. (2012) significantly showed that an acute aerobic exercise bout performed $6 \mathrm{~h}$ before power training enhanced the anabolic signaling compared with power training by itself. It should also be addressed that the total exercise volume of mice in the low-intensity exercise group and the high-intensity exercise group was different in the present study. Further experiments with matched total volume of exercise between low-intensity and high- intensity groups are warranted.

\section{Conclusions}

Our study showed that chronic low intensity aerobic training enhanced muscle growth, indicating that mild aerobic exercise play a role in maintaining muscle mass.

\section{Conflict of Interest}

There is no conflict of interest.

\section{Acknowledgements}

This study was supported in part by a Grant-in-Aid for Scientific Research from the Japanese Ministry of Education, Science, Sports, and Culture (grant No. 24500853).

\section{References}

BOLSTER DR, CROZIER SJ, KIMBALL SR, JEFFERSON LS: AMP-activated protein kinase suppresses protein synthesis in rat skeletal muscle through down-regulated mammalian target of rapamycin (mTOR) signaling. J Biol Chem 277: 23977-23980, 2002.

BURD NA, GORISSEN SH, VAN LOON LJ: Anabolic resistance of muscle protein synthesis with aging. Exerc Sport Sci Rev 41: 169-173, 2013.

CHO J, LEE I, KIM D, KOH Y, KONG J, LEE S, KANG H: Effect of aerobic exercise training on non-alcoholic fatty liver disease induced by a high fat diet in C57BL/6 mice. J Exerc Nutr Biochem 18: 339-346, 2014.

DICKINSON JM, VOLPI E, RASMUSSEN BB: Exercise and nutrition to target protein synthesis impairments in aging skeletal muscle. Exerc Sport Sci Rev 41: 216-223, 2013.

DURHAM WJ, CASPERSON SL, DILLON EL, KESKE MA, PADDON-JONES D, SANFORD AP, HICKNER RC, GRADY JJ, SHEFFIELD-MOORE M: Age-related anabolic resistance after endurance-type exercise in healthy humans. FASEB $J$ 24: 4117-4127, 2010.

EGAN B, ZIERATH JR: Exercise metabolism and the molecular regulation of skeletal muscle adaptation. Cell Metab 17: 162-184, 2013.

FUJITA S, RASMUSSEN BB, CADENAS JG, DRUMMOND MJ, GLYNN EL, SATTLER FR, VOLPI E: Aerobic exercise overcomes the age-related insulin resistance of muscle protein metabolism by improving endothelial function and Akt/mammalian target of rapamycin signaling. Diabetes 56: 1615-1622, 2007.

FYFE JJ, BISHOP DJ, STEPTO NK: Interference between concurrent resistance and endurance exercise: molecular bases and the role of individual training variables. Sports Med 44: 743-762, 2014.

GARBER CE, BLISSMER B, DESCHENES MR, FRANKLIN BA, LAMONTE MJ, LEE IM, NIEMAN DC, SWAIN DP, AMERICAN COLLEGE OF SPORTS MEDICINE: Quantity and quality of exercise for developing and maintaining cardiorespiratory, musculoskeletal, and neuromotor fitness in apparently healthy adults: guidance for prescribing exercise. Med Sci Sports Exerc 43: 1334-1359, 2011.

GORDON SE, FLUCK M, BOOTH FW: Selected contribution: Skeletal muscle focal adhesion kinase, paxillin, and serum response factor are loading dependent. $J$ Appl Physiol (1985) 90: 1174-1183; discussion 1165, 2001.

HARBER MP, KONOPKA AR, JEMIOLO B, TRAPPE SW, TRAPPE TA, REIDY PT: Muscle protein synthesis and gene expression during recovery from aerobic exercise in the fasted and fed states. Am J Physiol Regul Integr Comp Physiol 299: R1254-R1262, 2010. 
HARBER MP, CRANE JD, DICKINSON JM, JEMIOLO B, RAUR U, TRAPPE TA, TRAPPE SW: Protein synthesis and the expression of growth-related genes are altered by running in human vastus lateralis and soleus muscles. Am J Physiol Regul Integr Comp Physiol 296: R708-R714, 2009.

HARBER MP, KONOPKA AR, DOUGLASS MD, MINCHEV K, KAMMINSKY LA, TRAPPE TA, TRAPPE S: Aerobic exercise training improves whole muscle and single myofiber size and function in older women. Am J Physiol Regul Integr Comp Physiol 297: R1452-R1459, 2009.

HARBER MP, KONOPKA AR, UNDEM MK, HINKLEY JM, MINCHEV K, KAMINSKY LA, TRAPPE TA, TRAPPE S: Aerobic exercise training induces skeletal muscle hypertrophy and age-dependent adaptations in myofiber function in young and older men. J Appl Physiol (1985) 113: 1495-1504, 2012.

HOLECEK M, MICUDA S: Amino acid concentrations and protein metabolism of two types of rat skeletal muscle in postprandial state and after brief starvation. Physiol Res 66: 959-967, 2017.

JIANG HY, KOIKE T, LI P, WANG ZH, KAWATA Y, OSHIDA Y: Combined effects of short-term calorie restriction and exercise on insulin action in normal rats. Horm Metab Res 42: 950-954, 2010.

JUNG DY, KO HJ, LICHTMAN EI, LEE E, LAWTON E, ONG H, YU K, AZUMA Y, FRIEDLINE RH, LEE KW, KIM JK: Short-term weight loss attenuates local tissue inflammation and improves insulin sensitivity without affecting adipose inflammation in obese mice. Am J Physiol Endocrinol Metab 304: E964-E976, 2013.

KENNEDY BK, LAMMING DW: The mechanistic target of rapamycin: the grand conducTOR of metabolism and aging. Cell Metab 23: 990-1003, 2016.

KIM E, GORAKSHA-HICKS P, LI L, NEUFELD TP, GUAN KL: Regulation of TORC1 by Rag GTPases in nutrient response. Nat Cell Biol 10: 935-945, 2008.

KONOPKA AR, HARBER MP: Skeletal muscle hypertrophy after aerobic exercise training. Exerc Sport Sci Rev 42: 53-61, 2014.

KOOPMAN R, ZORENC AH, GRANSIER RJ, CAMERON-SMITH D, VAN LOON LJ: Increase in S6K1 phosphorylation in human skeletal muscle following resistance exercise occurs mainly in type II muscle fibers. Am J Physiol Endocrinol Metab 290: E1245-E1252, 2006.

KWON SM, PARK HG, JUN JK, LEE WL: Exercise, but not quercetin, ameliorates inflammation, mitochondrial biogenesis, and lipid metabolism in skeletal muscle after strenuous exercise by high-fat diet mice. J Exerc Nutr Biochem 18: 51-60, 2014.

LI P, KOIKE T, QIN B, KUBOTA M, KAWATA Y, JIA YJ, OSHIDA Y: A high-fructose diet impairs Akt and PKCzeta phosphorylation and GLUT 4 translocation in rat skeletal muscle. Horm Metab Res 40: 528-532, 2008.

LUNDBERG TR, FERNANDEZ-GONZALO R, GUSTAFSSON T, TESCH PA: Aerobic exercise alters skeletal muscle molecular responses to resistance exercise. Med Sci Sports Exerc 44: 1680-1688, 2012.

MAKANAE Y, KAWADA S, SASAKI K, NAKAZATO K, ISHII N: Vitamin C administration attenuates overloadinduced skeletal muscle hypertrophy in rats. Acta Physiol (Oxf) 208: 57-65, 2013.

MARCELL TJ: Sarcopenia: causes, consequences, and preventions. J Gerontol A Biol Sci Med Sci 58: M911-M916, 2003.

MUTHNY T, KOVARIK M, SISPERA L, TILSER I, HOLECEK M: Protein metabolism in slow- and fast-twitch skeletal muscle during turpentine-induced inflammation. Int J Exp Path 89: 64-71, 2008.

PETERSON MD, SEN A, GORDON PM: Influence of resistance exercise on lean body mass in aging adults: a metaanalysis. Med Sci Sports Exerc 43: 249-258, 2011.

RUDERMAN NB, CARLING D, PRENTKI M, CACICEDO JM: AMPK, insulin resistance, and the metabolic syndrome. J Clin Invest 123: 2764-2772, 2013.

SANDRI M: Autophagy in skeletal muscle. FEBS Lett 584: 1411-1416, 2010.

SANDRI M: Protein breakdown in muscle wasting: Role of autophagy-lysosome and ubiquitin-proteasome. Int J Biochem Cell Biol 45: 2121-2129, 2013.

SCHIAFFINO S, DYAR KA, CICILIOT S, BLAAUW B, SANDRI M: Mechanisms regulating skeletal muscle growth and atrophy. FEBS J 280: 4294-4314, 2013.

SERRANO AL, BAEZA-RAJA B, PERDIGUERO E, JARDI M, MUNOZ-CANOVES P: Interleukin-6 is an essential regulator of satellite cell-mediated skeletal muscle hypertrophy. Cell Metab 7: 33-44, 2008. 
SHORT KR, VITTONE JL, BIGELOW ML, PROCTOR DN, NAIR KS: Age and aerobic exercise training effects on whole body and muscle protein metabolism. Am J Physiol Endocrinol Metab 286: E92-E101, 2004.

SPANGENBURG EE: Changes in muscle mass with mechanical load: possible cellular mechanisms. Appl Physiol Nutr Metab 34: 328-335, 2009.

THOMSON DM, GORDON SE: Diminished overload-induced hypertrophy in aged fast-twitch skeletal muscle is associated with AMPK hyperphosphorylation. J Appl Physiol (1985) 98: 557-564, 2005.

YUAN H, NIU Y, LIU X, YANG F, NIU W, FU L: Proteomic analysis of skeletal muscle in insulin-resistant mice: Response to 6-week aerobic exercise. PLoS One 8: e53887, 2013.

XUE QL: The frailty syndrome: definition and natural history. Clin Geriatr Med 27: 1-15, 2011. 